\title{
A Novel Fabrication Technique for Developing Metal Nanodroplet Arrays
}

Christopher Edgar, Chad Johns, and M. Saif Islam

Electrical and Computer Engineering, University of California, Davis, One Shields Avenue, 1207 Kemper, Davis, California, 95616

\section{INTRODUCTION}

Metal in particle form is useful in fabrication of silicon nanowires [1], superlattice nanowires [2], colloidal solutions, biomedical applications [3,4], photonics [5] and sensors [6]. Incorporation of such research into manufacturing processes requires new research in fabrication processes for scalable and reproducible low cost arrays of nanoparticles. Exploiting known metallurgical sciences, new approaches in fabrication technology are constantly approaching a goal for the fabrication of oriented arrays of nanoparticles [7,8]. At present these existing processes are not capable of wide-scale deployment and selective array fabrication. In this paper, we demonstrate a novel process for ultimately developing well-defined arrays of metal nanodroplets that can be incorporated into most microfabrication processes.

\section{EXPERIMENTAL DETAILS}

First, a common silicon wafer was cleaned, dried, and soft-baked at $110^{\circ} \mathrm{C}$ for 60 seconds to remove residual water from the substrate. Photoresist (Shipley Corporation) was next applied and spun on and baked at $110^{\circ} \mathrm{C}$ to stabilize the photoresist for further processing. The photoresist was next exposed by a mask aligner (Karl-Suss) to produce patterned arrays of microstructures. The exposed substrate was then developed for 50 seconds, and then rinsed in de-ionized water and nitrogen dried. Metal deposition was performed in an electron-beam evaporation chamber (CHA) to control the thickness of the metal pattern lines. Our experiments also used angular deposition in the e-beam to produce smaller width metal patterned lines. Liftoff was performed and the sample was once again washed and nitrogen dried. The annealing process was carried out using a Vacuum Tube Furnace (MTI Crystal, GSL-1600X) at a temperature range of $400^{\circ} \mathrm{C}$ to $1000^{\circ} \mathrm{C}$ under the flow of argon gas. Characterization was performed by using a scanning electron microscope (FEI XL30-SFEG), and a digital camera optical microscopy station.

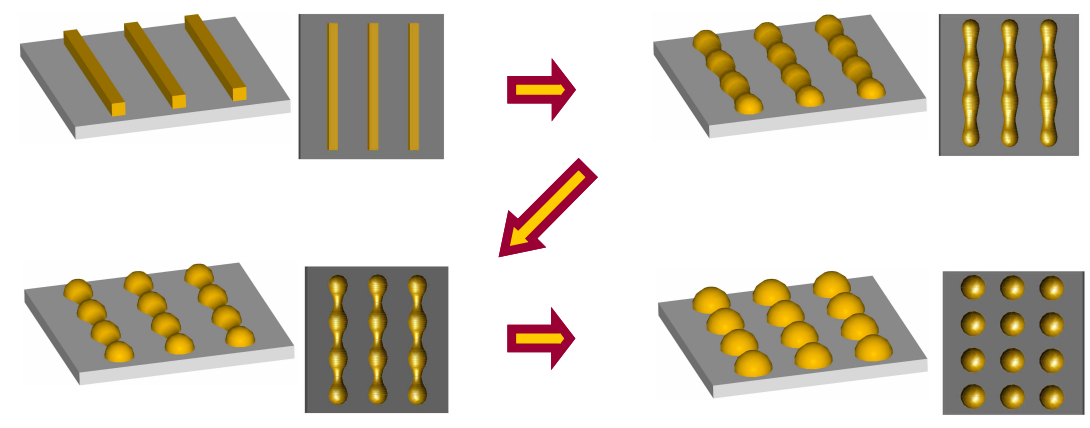

Figure 1 Schematic of ideal metal patterned lines that experience transformation from linear metal patterned lines into uniform isolated droplets in place of the original metal. 


\section{DISCUSSION}

Figure 1 shows a schematic representation of the process that is used to develop metal nanodroplet arrays. Metal when exposed to an elevated temperature becomes unstable and break down into metal clusters that eventually form into droplets. In our experiments, we have gold metal lines on a silicon substrate and the heating of the metal lines on the substrate causes diffusion of the silicon substrate atoms into the gold metal bulk, and gold atoms into the silicon substrate. The mixing of silicon atoms into the gold patterns allows for our method for the formation of droplet arrays. The melting temperature of the original bulk gold droplet decreases as gold atoms are replaced by silicon atoms. This allows our experiments to run successfully at temperatures below the recorded melting temperature of pure gold $\left(1084^{\circ} \mathrm{C}\right)$ [10]. In consideration of the droplet shape that is to form, the height of the metal line must be considered as well. In our experiments, we used e-beam metal evaporation to control our metal pattern thicknesses. Figure 2 shows an example of our proposed method of observing how metal droplets will form.

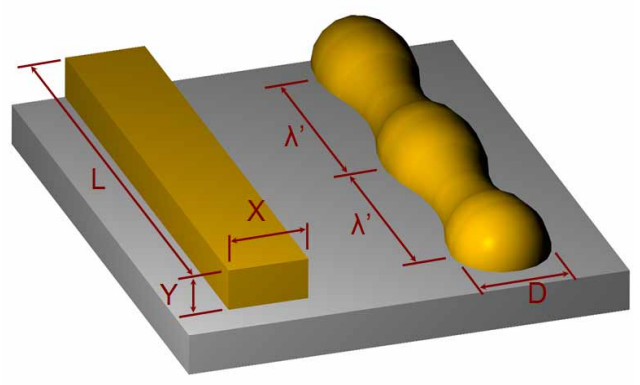

Figure 2 Consideration of the cross-sectional area of the original metal pattern for the formation of droplets. Adjusting the $\mathrm{X}$ and $\mathrm{Y}$ distances in our experiments shows droplets can form as a single strand or multiple particles adjacent in a linear array.

Figure 3 shows an array of nanodroplets on top of a silicon (100) substrate formed by our annealing technique. Nanodroplets have a diameter around $800 \mathrm{~nm}$ wide on the $1 \mu \mathrm{m}$ metal patterned line, where wider metal lines have multiple droplets in the array. Of significant importance is that the particle formation was in place of the patterned metal lines, which shows
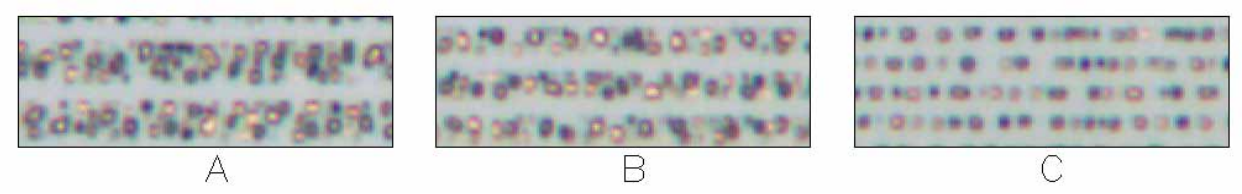

Figure 3 Optical microscope images showing top view of silicon (100) wafer with four sections of $100 \AA$ thick gold metal patterned lines all spaced $1 \mu \mathrm{m}$ apart. Sample was annealed at $800^{\circ} \mathrm{C}$ for 3 hours. A) $3 \mu \mathrm{m}$ wide gold lines broke down into droplets that are not linear. The width of the array is 3 droplets wide and shows the limits of where droplets can form. B) $2 \mu \mathrm{m}$ wide gold lines broke down into droplets that show mostly 2 droplets wide. Again, the width of the metal patterned line limits where droplets can form. C) $1 \mu \mathrm{m}$ wide gold lines broke down into linear droplets. Single droplet widths form along the length of the metal patterned line and are highly directional. 
that linear placement of the metal nanoparticles is possible by this technique if the height and the width of the metal pattern is controlled. The breakdown of the metal into an unstable state was induced by annealing the metal and substrate and particles formed in a manner similar to that of Rayleigh Instability [9]. Since these experiments were conducted at $800^{\circ} \mathrm{C}$, the silicon-gold composition range present in each droplet can be very random.

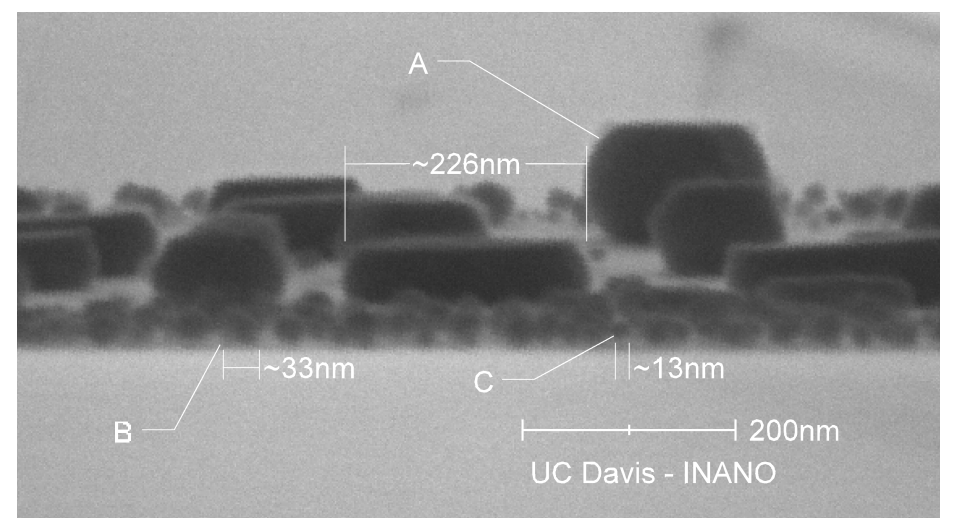

Figure 4: SEM showing angled view of particles formed from gold metal lines. Metal widths range from $5 \mu \mathrm{m}$ wide to $1 \mu \mathrm{m}$ wide and all are $150 \AA$ thick. The larger droplets have a flattened top and the smaller particles are a visibly completed sphere. A) Larger nanoscale particles form as continuous heating causes smaller droplets to move and combine to form larger droplets. B \& C) Smaller diameter droplets formed from the $1 \mu \mathrm{m}$ wide metal lines.

Figure 4 shows an image where SEM was performed on a silicon wafer with $150 \AA$ thick gold lines that was annealed for 2 hours. Some of the particles in the array of Figure 2 are noticeably much smaller than their neighbor particles. This is possibly due to the formation of metal patterns not having a good height and width ratios that make up the overall volume of the metal structure prior to annealing. This may also be caused by more of the volume along the linear
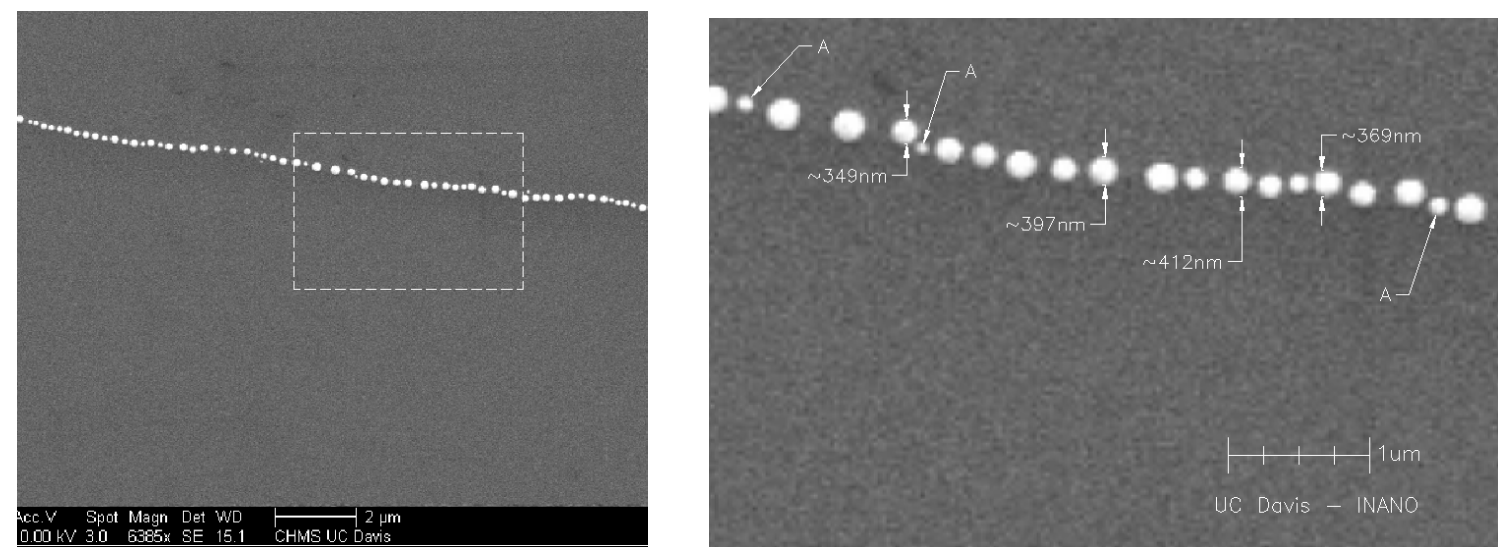

Figure 5 An SEM image of an approximately 400nm wide by $200 \AA$ thick gold metal film produced from angular deposition annealed at $800^{\circ} \mathrm{C}$ for 3 hours. Angular deposition could not produce perfectly linear patterned lines but this experiment is to show reducing the metal pattern thickness and width can control the size of the droplets formed. The film broke down into an array of nanoscale droplets possessing an average diameter around $381 \mathrm{~nm}$ with a few smaller droplets (A) that exist due to the slightly irregular original metal pattern. 
metal pattern to move to the larger forming droplets and the remaining metal not in transport remains and forms smaller particles. Visibly, the larger particles exhibit flattened tops resulting in partially formed droplets where the smaller droplets appear spherical. This can be attributed to the insufficient number of atoms in the larger particles for the completion of the sphere. The smaller particles therefore have enough atoms to complete the sphere.

Figure 5 shows a particle array formed from annealing a patterned 400nm wide having approximately $200 \AA$ thick gold line created using angular deposition. Since these droplets are formed along a rough linear direction having a slight curvature, it can be seen that curved metal patterns can produce curved droplet arrays. The droplets that form are closely sized the same and are isolated from each other with the existence of few smaller particles. Again, we explored the possible width-height ratio of the metal pattern that would eventually form into droplets where these results show clearly more control of the size of the particles by reducing the width of the original metal patterned line.

While at the gold-silicon substrate interface, gradual diffusion and mixing of the two elements causes the droplet to stick to the substrate when the droplet forms. Extensive chemical analysis of these particles is needed to derive the mechanics that drive the droplet formation by this technique. Removal of these droplets by scraping the sample concluded that dislodging the particles can be accomplished, but some of the particle can remain once the bulk droplet is removed. The edges of individual gold-silicon droplet also exhibit random wetting angles when in contact with the substrate. Since gold and silicon do not crystallize and silicon can oxidize, the different wetting angles observed we theorize as the differing compositions that can exist for the silicon in gold and the presence of $\mathrm{SiO}_{2}$ in the droplet as well during the experiment. Future experimentation on such particles is needed to accurately verify the above theory.

\section{CONCLUSIONS}

We have successfully demonstrated a novel scalable metal nanodroplet array manufacturing technique applicable into most clean room fabrication processes. The implication of this technique only requires minor adjustment to clean room processes by considering an annealing stage. This approach produces metal droplets into a self-assembly technique directly influenced by the initial width and height of the metal pattern on the substrate. Smaller width and height metal patterns allow for smaller diameter single linear droplets to form. Although the droplets shown in the above images form where they set at random distances apart, more accurate positioning and creating uniform droplets in future experiments may be accomplished by controlling the metal grains present, the shape of the metal patterns prior to annealing, using varying metals and compositions, or even the annealing environment used (temperature, time, etc). This technique of forming regular arrays of metal nano-particles can offer exciting opportunities in plasmonics, bio-chemical sensing and ultra-high density information storage and can be further developed into metal-catalyzed nanowires with uniform diameters and spacing offering exciting opportunities for precise positioning of nanowires. Furthermore, the prospect of using metal nanoparticle chains to promulgate optical excitations is attractive for integrated optics applications, since it can lead to optical processing circuitry with dimensions equivalent to the wavelength of the light. 


\section{REFERENCES}

1. R. S Wagner, and C. S. Elliot, App. Phy. Let. 4, 89 (1964).

2. M. S. Gudiksen, L. J. Lauhon, J. Wang, D. C. Smith, C. M. Lieber, Nature 415, 617 (2002).

3. J. Zhang, J. R. Lakowicz, J. Phys Chem. B 2006, 2387 (2006).

4. Q. Wang, H. Yu, L. Zhong, J. Liu, J. Sun, J. Shen, Chem. Mater. 18, 1988 (2006).

5. A. K. Sharma, B. D. Gupta, Elsevier Photonics and Nanostructures - Fundamentals and App. 3, 30 (2005).

6. G. Bauer, F. Pittner, Th. Schalkhammer, Mikrochimica Acta 131, 107 (1999).

7. B. Sun, R. H. Friend, H. Guo, D. Nau, H. Giessen, X. Zhang, Nano Lett. 6, 651 (2006).

8. J. Huang, A. R. Tao, S. Connor, R. He, P. Yang, Nano Lett. 6, 524 (2006).

9. A. M. Glaeser, Interface Science 9, 65 (2001).

10. Alloy Phase Diagrams, ASM Handbook, Materials Park, OH, 3 (1992). 